NISTIR 5019

PROPOSED UNCERTAINTY ANALYSIS FOR RCS MEASUREMENTS

R.C. Wittmann

M.H. Francis

L.A. Muth

R.L. Lewis

QC

100

.056

N0.5019

1994 



\section{PROPOSED UNCERTAINTY ANALYSIS FOR RCS MEASUREMENTS}

\section{R.C. Wittmann}

M.H. Francis

L.A. Muth

R.L. Lewis

\section{Electromagnetic Fields Division}

Electronics and Electrical Engineering Laboratory

National Institute of Standards and Technology

Boulder, Colorado 80303-3328

January 1994

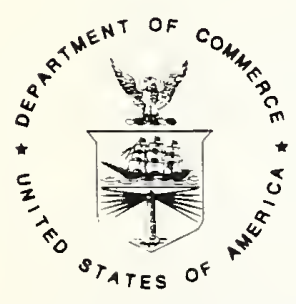

U.S. DEPARTMENT OF COMMERCE, Ronald H. Brown, Secretary TECHNOLOGY ADMINISTRATION, Mary Good, Under Secretary for Technology NATIONAL INSTITUTE OF STANDARDS AND TECHNOLOGY, Arati Prabhakar, Director 


\title{
Proposed Uncertainty Analysis for RCS Measurements
}

\author{
R. C. Wittmann M. H. Francis L. A. Muth \\ R. L. Lewis
}

\begin{abstract}
From a study of several Radar Cross Section (RCS) measurement facilities, we identify significant sources of uncertainty and develop methods for estimating their effect. Our goal is to provide a reasonable and uniform formalism for evaluating RCS measurements which can be used on a variety of test ranges to produce comparable estimates of uncertainty.
\end{abstract}

\section{Introduction}

All measurements of a quantity, such as $\sigma$ (Radar Cross Section or RCS), should be quantified by a statement of uncertainty

$$
\sigma=\sigma_{0} \pm \Delta \sigma,
$$

which indicates the metrologists's best estimate $\sigma_{0}$ and an uncertainty $\Delta \sigma>$ 0 which is suggested as a reasonable bound for the measurement error. In addition, uncertainty estimation often identifies the principal sources of error which must be must be controlled to improve measurement quality. The intent of this report is to provide an outline which may serve as a basis for RCS uncertainty analysis.

Our use of the adjective "reasonable" is intentionally imprecise (see appendix A). As will become apparent, determination of $\Delta \sigma$ is often subjective. It is therefore of paramount importance to clearly delineate assumptions and procedures leading to the estimate of uncertainty. The end user should be able to apply this information to reach his or her own conclusions about the quality of the data. Furthermore, in areas (such as RCS) where many workers use a variety of techniques to measure a certain parameter, it is of value to have a common method for estimating uncertainty. When a uniform approach is used, reported uncertainties better reflect the relative merit of corresponding measurements. 
Although we might wish otherwise, the procedures presented here will not cover all possibilities. It will be necessary to correct, modify, or develop new approaches to handle specific measurement problems. As always, the final responsibility for a meaningful uncertainty analysis rests with the metrologist.

Section 2 presents an overview of the proposed uncertainty analysis, which is a hierarchical system of tables for reporting and summarizing components of uncertainty. The individual components of uncertainty are described in section 3 . Here we identify sources of uncertainty and discuss methods for estimating their impact on RCS measurement errors. The discussion is general, and modification may be required for application to an individual range. Section 4, which parallels section 3, discusses uncertainties associated with measurement of the calibration target.

\section{Reporting Measurement Uncertainty}

Error bounds need not be symmetric:

$$
\sigma_{0}+\Delta \sigma_{+}>\sigma>\sigma_{0}-\Delta \sigma_{-} .
$$

For simplicity, we will continue to use eq (1) with $\Delta \sigma=\max \left(\Delta \sigma_{+}, \Delta \sigma_{-}\right)$.

Uncertainties are also stated logarithmically

$$
\Delta \sigma_{ \pm}(\mathrm{dB})=10 \log \left(1 \pm \frac{\Delta \sigma}{\sigma_{0}}\right)
$$

As before, we will often use symmetric bounds. In this case

$$
\Delta \sigma(\mathrm{dB}) \equiv-\Delta \sigma_{-}(\mathrm{dB})=-10 \log \left(1-\frac{\Delta \sigma}{\sigma_{0}}\right) .
$$

However, because of the nonlinear nature of eq (3) unsymmetrical bounds may be given when reporting larger logarithmic uncertainties. For example, if $\Delta \sigma / \sigma_{0}=1$, then $\Delta \sigma_{+}(\mathrm{dB}) \approx 3$, while $\Delta \sigma_{-}(\mathrm{dB})=-\infty$. In the limit $\Delta \sigma \rightarrow 0$, logarithmic uncertainty is linearly related to relative uncertainty by

$$
\Delta \sigma(\mathrm{dB}) \approx 4.34 \frac{\Delta \sigma}{\sigma_{0}} .
$$

Sample uncertainty tables are shown in figure 1 . The numbers to the left of each entry are references to the section of this report where the corresponding uncertainty is discussed. Individual sources of uncertainty (called components of uncertainty) are listed above the line in each table. These are combined to give overall estimates of uncertainty which are shown below the lines. Uncertainties are reported logarithmically. An entry of "neg." 
3.1 Average Illumination $\quad 0.4$

3.2 Background-Target 0.1 Interactions

3.3 Cross Polarization 0.6

$\begin{array}{lll}3.4 & \text { Drift } & 1.0\end{array}$

3.5 Frequency neg.

3.6 Integration neg.

3.7 I-Q Imbalance neg.

3.8 Near Field 1.0

3.9 Noise-Background $\quad 0.9$

3.10 Nonlinearity $\quad 1.0$

3.11 Range neg.

3.12 Target Orientation n.a.

$\begin{array}{ll}3.13 \text { Calibration Target (4.14) } & 0.9 \\ 3.14 & \text { Overall Uncertainty (RSS) }\end{array}$

\section{CALIBRATION TARGET UNCERTAINTIES}

4.1 Average Illumination

0.0

4.2 Background-Target

0.1

Interactions

4.3 Cross Polarization $\quad 0.0$

4.4 Drift neg.

4.5 Frequency neg.

4.6 Integration n.a.

4.7 I-Q Imbalance neg.

4.8 Near Field neg.

4.9 Noise-Background 0.9

4.10 Nonlinearity $\quad 0.0$

4.11 Range neg.

4.12 Target Orientation $\quad 0.0$

4.13 Calibration Target $\quad 0.1$

4.14 Overall Uncertainty (RSS)

0.9

Figure 1: A sample summary of RCS uncertainties. 
indicates that the effect has negligible impact (here taken operationally as less than $0.1 \mathrm{~dB}$ ). An entry of "n.a." indicates that this source is not considered a factor in the current evaluation (integration error, for example, is not applicable when the target is stationary). The entries are arbitrary and do not necessarily represent typical uncertainties.

The method of uncertainty combination is Root Sum Square (RSS). That is, the overall uncertainty $\Delta \sigma$ is calculated as

$$
\left(\frac{\Delta \sigma}{\sigma_{0}}\right)^{2}=\sum_{i}\left(\frac{\Delta \sigma_{i}}{\sigma_{0}}\right)^{2},
$$

where $\Delta \sigma_{i}$ are the components of uncertainty. Note that relative (not logarithmic) uncertainty is used in the calculation of eq (6).

In turn, each component of uncertainty may be the overall uncertainty of a separate lower level table. An example is the second table in figure 1 , which gives uncertainties associated with the standard target calibration. The overall calibration target uncertainty (4.14) is included above as a contribution to the measurement uncertainty (3.13). The upper level tables presented in figure 1 provide a convenient summary of the major features of the analysis. Lower level tables may not be formally provided but they should be available to document the details. Contributions which are typically subdivided include noise and background (here combined in 3.9) and nonlinearity effects (3.10) which are often specified on a component by component basis.

The uncertainty analysis may be compared to the roots of a tree, expanding downward in ever greater detail. At the lowest level we use worst-case estimates of uncertainty so that each component is an RSS of one or more worst-case uncertainties.

The term "worst case" is applied subjectively. Estimates of worst-case uncertainty will generally be strongly influenced by practical experience. This is especially true for uncertainties which depend on the nature of the target, such as those associated with cross-polarization errors. In cases where the uncertainty in a parameter has been estimated statistically, we will (by fiat) equate the corresponding worst-case uncertainty with two standard deviations. (For example, assume that the ruler used in an experiment comes from a lot with a standard deviation in length of $1 \mathrm{~mm}$. We would assign a worst-case length uncertainty of $2 \mathrm{~mm}$.)

Uncertainty tables, such as figure 1 , must be valid over the entire range of parameters which occur in a given experiment. When uncertainty is a strong function of a parameter (such as signal-to-noise ratio) it may be desirable to divide the parameter range into parts and produce separate uncertainty tables for each. Thus, there could be a table for peak signals, where the signal-to-noise ratio is most favorable, and another table for uncertainty in 
sidelobe levels. Similarly it may be advantageous to report uncertainty as a function of frequency band.

\section{Unknown Target Uncertainties}

Following current practice, the scope of this report is largely limited to a discussion of monostatic measurement of scalar, main-polarization RCS, that is, to the measurement of the squared amplitude of the $v v$ or $h h$ component of the scattering matrix ( $v$ for vertical, $h$ for horizontal polarization). For far-field measurement the governing relationship is the radar equation, here expressed as the ratio of unknown target RCS to calibration target RCS:

$$
\frac{\sigma_{0}}{\sigma_{s}}=\left(\frac{R}{R_{s}}\right)^{4}\left(\frac{G_{s}}{G}\right)^{2}\left(\frac{f}{f_{s}}\right)^{2} \frac{P_{s t}}{P_{t}} \frac{P_{r}}{P_{s r}}
$$

where

$$
\begin{aligned}
\sigma & =\text { radar cross section } m^{2} \\
R & =\text { range (distance) } m \\
G & =\text { antenna gain, } \\
f & =\text { frequency } s^{-1} \\
P_{t} & =\text { transmitted power } W \\
P_{r} & =\text { received power } W
\end{aligned}
$$

The subscript $s$ identifies quantities associated with the (standard) calibration target. Since eq ') does not explicitly apply, compact range measurements are given separate consideration where appropriate.

To simplify notation where the context allows, we will use the symbol $\Delta \sigma$ to denote a generic component of uncertainty. For simplicity, first-order calculations are often employed, even when uncertainties are large. (More accurate computation may not be justified in some cases, due to highly subjective evaluations in other cases.)

\subsection{Average lllumination}

Here we discuss uncertainties due to geometry that result from differences in average illumination between unknown target and calibration target measurements.

Dynamic range pointing errors introduce an uncertainty in RCS through gain reduction. Let

$$
\frac{G}{G_{0}}=\cos ^{2}\left(\frac{\pi \theta}{4 \theta_{0}}\right),
$$

where $\theta_{0}$ is $1 / 2$ of the antenna's 3 - $\mathrm{dB}$ beamwidth, $\theta$ is the worst-case pointing error, and $G / G_{0}$ is the gain reduction factor. Then from eq (7) 


$$
\Delta \sigma(\mathrm{dB})=-40 \log \left[\cos \left(\frac{\pi \theta}{4 \theta_{0}}\right)\right] .
$$

Equation (9) assumes that the antenna has a $\cos ^{2}$ pattern and is optimally boresighted. Any valid approximation is acceptable, although measured pattern data are preferred. Estimation of tracking uncertainty by including contributions from both eqs (9) and (20) is, perhaps, overly conservative. A closer examination indicates that, at least to first order, tracking error is bounded by the larger of the values eqs (9) and (20). The table entry corresponding to the smaller value may be set to 0 .

For static and compact ranges, illumination uncertainty is not so much a matter of pointing, but rather is tied to the fact that illumination is nonuniform over the test volume. Let us assume that the incident field can be divided into an ideal plane wave plus a residual nonuniform component. The impact of the nonuniform component is considered in section 3.8. It is possible to correct for average illumination effects by determining $G_{s} / G \equiv\left\langle E_{s}^{2}\right\rangle /\left\langle E^{2}\right\rangle$ [see eq (7)] from measured or calculated field data. The average intensities $\left\langle E_{s}^{2}\right\rangle$ and $\left\langle E^{2}\right\rangle$ are computed over the calibration target and unknown target volumes, respectively. Uncertainty in $G_{s} / G$ should be estimated and entered here. For example, a relative gain uncertainty of 0.1 $\mathrm{dB}$ produces a component of uncertainty $\Delta \sigma \approx 0.2 \mathrm{~dB}$.

\subsection{Background-Target Interactions}

This effect concerns energy that at some point is scattered from the target and then rescattered by the background. On static ranges, this is primarily a problem of target-support interaction. A thorough analytic treatment of this interaction seems impractical at best. The effect could be studied experimentally by varying the target-support interface. Currently, estimates of this uncertainty should be regarded as especially tenuous.

\subsection{Cross Polarization}

Here we account for the fact that due to alignment or physical imperfection, the antenna will not be perfectly polarized ( $h$ or $v$ ). If a target's crosspolarization response is large compared to the main-polarization response, there can be significant error even if the antenna has good polarization isolation.

Define the scattering matrix parameters

$$
\begin{array}{ll}
R_{\mu} & \text { receiving (antenna), } \\
S_{\mu} \quad \text { transmitting (antenna), } \\
T_{\nu \mu} \text { scattering (target), }
\end{array}
$$


where $\nu$ and $\mu$ are $v$ for vertical polarization and $h$ for horizontal polarization. The received signal is

$$
S=R_{v} T_{v v} S_{v}+R_{v} T_{v h} S_{h}+R_{h} T_{h v} S_{v}+R_{h} T_{h h} S_{h}
$$

or

$$
S=R_{v} T_{v v} R_{v}+2 R_{v} T_{v h} R_{h}+R_{h} T_{h h} R_{h}
$$

for a reciprocal monostatic measurement system. Consider the case of a vertically polarized antenna, so $R_{v} \gg R_{h}$. The first term on the right of eq $(11)$ is the desired interaction $(v v)$, the second term $(v h)$ is a first-order error, and the third term $(h h)$ is a second-order error. We will model the unknown target as strongly depolarizing with $T_{v v}=T_{h h}=T_{v h}$, or

$$
\left|\frac{\Delta S}{S}\right| \approx 2\left|\frac{R_{h}}{R_{v}}\right|
$$

Thus,

$$
\Delta \sigma(\mathrm{dB})=-20 \log \left(1-2 \times 10^{-\epsilon_{p} / 20}\right),
$$

where $\epsilon_{p}$ is the antenna polarization isolation. [In this case $\epsilon_{p}=20 \log \left(R_{v} / R_{h}\right)$.] It is important to remember that eq (13) makes specific assumptions about the unknown target and does not represent the worst-case scenario. As an example, according to eq (13) a polarization isolation of $30 \mathrm{~dB}$ gives a component of uncertainty of $0.6 \mathrm{~dB}$.

In principle, it is possible to correct for polarization errors if complete polarimetric measurements were available [ 1 , for example].

\subsection{Drift}

All physical systems are unstable to a certain degree. Drift uncertainty can be explored by observing a fixed target over an extended time. During a measurement, drift may be checked by observing a secondary standard, or by periodically remeasuring a particular target orientation. In fact it is possible to correct for drift by adjusting the system gain after each drift check. The component of uncertainty may be given as the expected drift over the duration of the measurement, or as the drift which is observed between successive calibration points. As an example, assume that extensive observation indicates that a measurement system drifts by less than $0.1 \mathrm{~dB}$ in an hour. An estimate of drift uncertainty for a half-hour measurement might be $0.05 \mathrm{~dB}$. 


\subsection{Frequency}

From eq (7) the component of uncertainty in RCS due to uncertainty in frequency is

$$
\Delta \sigma(\mathrm{dB})=-20 \log \left(1-\frac{\Delta f}{f}\right) .
$$

The uncertainty $\Delta f$ in frequency may be taken as the effective bandwidth of the system, that is, as the minimum of the transmitted bandwidth and the received bandwidth. (Where software gating is applied, it is necessary to consider the effect of digital filtering in estimating $\Delta f$.) For example, consider a measurement at $10 \mathrm{GHz}$ with a transmitted bandwidth of 20 $\mathrm{MHz}$ (corresponding to a $50 \mathrm{~ns}$ pulse). Then, $\Delta f \leq 20 \mathrm{MHz}$ and $\Delta \sigma(\mathrm{dB})$ $=0.02 \mathrm{~dB}$.

Frequency uncertainty is easy to control and should usually be negligible. If this uncertainty were important, we should consider, in addition to eq (14), that unknown target RCS, calibration target RCS, and system gain all depend implicitly on frequency (see appendix C).

\subsection{Integration (Moving Target)}

This uncertainty is due to target motion during the measurement. On static ranges, integration error can be controlled by slowing or stopping target rotation, or by doing less averaging. On dynamic ranges such control is much more difficult. Perhaps the best way to estimate this target dependent effect is through simulation.

\subsection{I-Q Imbalance}

For an input test signal $\cos (\omega t+\phi)$, an amplitude imbalance $\alpha$, and a phase imbalance $\beta$, the in-phase and quadrature responses of the receiving system (see section 3.10) are

$$
\begin{gathered}
I=\kappa \cos (\phi), \\
Q=\alpha \kappa \sin (\phi-\beta) .
\end{gathered}
$$

(The constant $\kappa$ is determined in the calibration.) When there is an I$\mathrm{Q}$ imbalance, measured amplitude will be a function of input phase. The uncertainty may be estimated by measuring power $\left[\propto\left(I^{2}+Q^{2}\right)\right]$ as input phase $\phi$ of the test signal is varied from $0^{\circ}$ to $360^{\circ}$. A peak-to-peak variation of $0.1 \mathrm{~dB}$, say, corresponds to about a $0.05 \mathrm{~dB}$ uncertainty in RCS.

\subsection{Near Field}

The radar equation, eq ( 7$)$, assumes that the target is illuminated by a plane wave. Compact ranges also try to approximate an incident plane wave. 
In practice, there will always be some taper or ripple. Complete analysis of illumination effects would be difficult and, perhaps, not very useful. A simple, but crude, estimate is to use peak-to-peak amplitude variation over the target volume (down range as well as cross range). That is, a $0.5 \mathrm{~dB}$ taper would give a $0.5 \mathrm{~dB}$ component RCS uncertainty. Although this estimate might be regarded as conservative for peak signals, the effect can be much larger for lower signals since nonideal illumination may cause locations of nulls to shift.

Our estimation method is most appropriate for targets whose RCS is dominated by a localized scatterer which might lie in a region of low or high illumination. Information on the location of target scattering centers can be used to refine uncertainty estimates. For example, if the primary scattering centers are relatively uniformly illuminated, then uncertainty may be reduced accordingly.

\subsection{Noise-Background}

Here we group sources of uncertainty which contribute to the received signal whether or not the target is present. Our estimation is by direct measurement with target absent and any support structure properly terminated. We employ background subtraction and averaging as appropriate to mitigate uncertainty. The observed residual noise-background is bounded by the worst-case estimate $N$. For a signal $S$, uncertainty is calculated as

$$
\Delta \sigma(\mathrm{dB})=-20 \log \left(1-10^{-\epsilon_{n} / 20}\right),
$$

where $\epsilon_{n}=20 \log (S / N)$. For a signal-to-noise ratio $\epsilon_{n}=20 \mathrm{~dB}, \Delta \sigma(\mathrm{dB})$ $\approx 1 \mathrm{~dB}$.

In some cases it might be preferable to estimate clutter and noise uncertainties separately and to enter their RSS here.

\subsection{Nonlinearity}

Assume that it is possible to break the rf feed between antenna and receiver so that a test signal can be injected into the "receiving system." The receiving system should include receiver, mixers, and as much of the rf hardware as possible while excluding the antenna. Significant sources of nonlinearity uncertainty which cannot be included in the receiving system (such as a remote mixer) must be separately evaluated (perhaps in a subtable).

When a complex signal $s=x+i y$ is injected into the receiving system, the response is given generally by

$$
s_{m}=K_{x}(x, y) x+i K_{y}(x, y) y+b
$$


When $K_{x} \neq K_{y}$, the system is imbalanced (see section 3.7). When $b \neq 0$, the system is biased (see section 3.9). When $K_{x}$ and $K_{y}$ depend on the input signal ( $x$ and $y$ ), the system is nonlinear. We assume that the receiving system is nearly ideal (after calibration) and that interaction between balance, bias, and linearity can be ignored to first order.

Nonlinearity uncertainty may be estimated by noting the residual deviation from linearity while using a precision attenuator to vary the test signal from a given reference level. The calibration target signal should be used as the reference level, if possible. Nonlinearity uncertainty can be no less than the calibration uncertainty of the precision attenuator.

\subsection{Range}

The uncertainty

$$
\Delta \sigma(\mathrm{dB})=-40 \log \left(1-\frac{\Delta R}{R}\right)
$$

is based on eq (7). In general, range can be measured accurately and accordingly range uncertainty is seldom significant.

Range uncertainty is not applicable (n.a.) to compact ranges.

\subsection{Target Orientation}

Uncertainty in orientation can lead to large errors, especially for electrically large objects. Uncertainty can be estimated as follows

$$
\Delta \sigma(\mathrm{dB})=-10 \log \left(1-\frac{\partial \sigma}{\partial \theta} \frac{\Delta \theta}{\sigma}\right),
$$

where $\sigma$ could be the predicted or, perhaps, the measured RCS, and $\theta$ is a suitable angular variable. If it is important to determine only peak and sidelobe envelope levels (which seems often the case), then this uncertainty can be largely ignored (n.a.).

In cases where orientation errors are significant, the process of binning (that is, averaging RCS over specified angular regions) can be useful; however, the bin size should be much larger than the uncertainty in orientation.

\subsection{Calibration Target}

The entry here is the overall uncertainty associated with measurement of the calibration target. See section 4.14. 


\subsection{Overall Uncertainty (RSS)}

The entry here is the RSS of the components of uncertainty sections 3.1-3.13. See eqs (3) and (6).

\section{Calibration Target Uncertainties}

The calibration target functions as a transfer standard to provide an absolute power reference for RCS measurements. The uncertainties associated with calibration are largely the same as those associated with any other RCS measurement. Thus, this section closely parallels section 3, but with additional comments on issues specific to calibration. (See appendix B for an alternate treatment of calibration uncertainties which reflects current practice at some dynamic ranges.)

\subsection{Average Illumination}

See section 3.1.

When the calibration target is tracked, we may compute a pointing-error uncertainty according to

$$
\Delta \sigma(\mathrm{dB})=-40 \log \left[\cos \left(\frac{\pi \theta_{s}}{4 \theta_{0}}\right)\right]
$$

When the calibration target is stationary we may use the (average) gain over the standard as the reference level and enter 0 for the component of uncertainty.

\subsection{Background-Target Interactions}

See the discussion in section 3.2 .

\subsection{Cross Polarization}

If we can assume that the calibration target is not depolarizing when it is properly aligned, we may take $T_{v v}=T_{h h}, T_{h v}=0$ in eq (11) to get (for a measurement of the $v v$ component)

$$
\left|\frac{\Delta S}{S}\right| \approx\left|\frac{R_{h}}{R_{v}}\right|^{2},
$$

which is basically a second-order effect. Thus,

$$
\Delta \sigma(\mathrm{dB})=-20 \log \left(1-10^{-\epsilon_{p} / 10}\right),
$$


where $\epsilon_{p}$ is the antenna polarization isolation (see section 3.3). Equation (22) makes specific assumptions about the calibration target, so it should be used with care.

As an example, according to eq (22) a polarization isolation of $30 \mathrm{~dB}$ gives a component of uncertainty of about $0.01 \mathrm{~dB}$.

\subsection{Drift}

See the discussion in section 3.4 .

\subsection{Frequency}

See the discussion in section 3.5 .

$$
\Delta \sigma(\mathrm{dB})=-20 \log \left(1-\frac{\Delta f_{s}}{f_{s}}\right) .
$$

\subsection{Integration}

See the discussion in section 3.6.

\subsection{I-Q Imbalance}

See the discussion in section 3.7.

\subsection{Near Field}

See the discussion in section 3.8 .

\subsection{Noise-Background}

See the discussion in section 3.9 .

$$
\Delta \sigma(\mathrm{dB})=-20 \log \left(1-10^{-\epsilon_{n} / 20}\right) .
$$

\subsection{Nonlinearity}

See the discussion in section 3.10. When the calibration target signal is used as the nonlinearity reference level, enter an uncertainty of 0 . Nonzero uncertainties are appropriate if the calibration level is not the same as the nonlinearity reference point or if the calibration target is measured over a wide range of levels, as in dynamic RCS calibrations where the calibration target is tracked as it moves through the range. 


\subsection{Range}

See the discussion in section 3.11 .

$$
\Delta \sigma(\mathrm{dB})=-40 \log \left(1-\frac{\Delta R_{s}}{R_{s}}\right) .
$$

\subsection{Target Orientation}

See the discussion in section 3.12 .

$$
\Delta \sigma(\mathrm{dB})=-10 \log \left(1-\frac{\partial \sigma_{s}}{\partial \theta_{s}} \frac{\Delta \theta_{s}}{\sigma_{s}}\right) .
$$

\subsection{Calibration Target}

This uncertainty addresses how well the RCS of the calibration target is known. Consider two cases:

(1) Primary Standard: For our purposes, this is a target whose RCS is computed. Errors arise because the predicted RCS value may be incorrect and/or because the actual standard object may correspond imperfectly to the ideal object. Uncertainty estimates usually range from negligib: for spheres, which make ideal primary standards, to several tenths of a decibel for plates and polyhedrals. Further research is needed to better evaluate this uncertainty.

(2) Transfer Standard: The RCS of such a calibration target is determined by comparison with another (base) standard. The uncertainty should be the overall uncertainty corresponding to (4.14) in a lower level table for the base standard.

\subsection{Overall Uncertainty (RSS)}

The entry here is the RSS of the components of uncertainty sections 4.1-4.13. See eqs (4) and (6).

\section{Acknowledgments}

We thank the following NIST personnel for helpful discussions an 1 suggestions during the preparation of this document: B.K. Alpert, D.A. Hill, R.M. Judish, A.G. Repjar, D. F.Vecchia, and J.C.M. Wang. 


\section{A Appendix Interpretation of Uncertainty}

In the main text we have presented a formalism for uncertainty analysis. Here we briefly discuss the motivation for our procedure, especially regarding guidelines presented in a recent NIST publication [2].

Uncertainties are commonly divided into the categories "random" and "systematic." Unfortunately, there seems to be no clear practical distinction between random and systematic, and we have made little attempt to distinguish these types of uncertainties in the main body of this report. We assume that any appropriate actions (such as averaging and background subtraction) already have been taken to mitigate uncertainty. The remaining uncertainties are evaluated as Type B uncertainties.

The treatment of Type B uncertainty in [2] is statistically motivated and is based on a generalized concept of probability [3]. The experimenter assigns a (prior) distribution for each parameter reflecting his or her "personal degree of belief" that the parameter might have some value. We may then apply a probabilistic analysis to estimate combined uncertainty. In this approach, it is natural to specify uncertainty in terms of probability, but this probability must be interpreted as a subjective belief and cannot (and must not) be interpreted in the usual random-variable (frequency) sense. To avoid confusion, we have de-emphasized probability, and have simply used the term "reasonable." This practice appropriately begs the question "just what is reasonable, anyway?"

Let us assume that a worst-case uncertainty is equivalent to a 2 -sigma uncertainty. [We write out sigma (standard deviation) to distinguish it from $\sigma$ (RCS).] That is, at least for a normal distribution, we are $95 \%$ certain that the error is bounded by our worst-case estimate. Under this hypothesis, the procedure of [2] is essentially identical to ours. However, the NIST document recommends reporting 1-sigma bounds for components of uncertainty and 2sigma bounds for expanded (overall) uncertainties. In this report we have used 2-sigma bounds for both component and overall uncertainties. Figure 2 is a modification of figure 1 following the NIST convention (with component of uncertainty $=\Delta \sigma / 2$ and overall uncertainty $=\Delta \sigma$ ). Note that the entries 4.14 and 3.13 are not the same since 4.14 is a 2 -sigma value and 3.13 is a 1 -sigma value. 
3.1 Average Illumination $\quad 0.2$

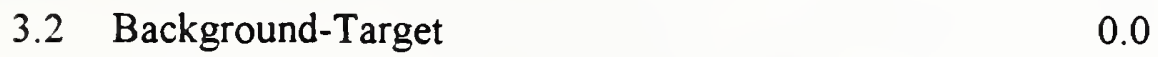
Interactions

3.3 Cross Polarization $\quad 0.3$

3.4 Drift 0.5

3.5 Frequency neg.

3.6 Integration neg.

3.7 I-Q Imbalance neg.

3.8 Near Field 0.5

3.9 Noise-Background 0.4

3.10 Nonlinearity $\quad 0.5$

3.11 Range neg.

3.12 Target Orientation n.a.

3.13 Calibration Target (4.14) $\quad 0.4$

3.14 Overall Uncertainty (RSS) $\quad 1.7$

\section{CALIBRATION TARGET UNCERTAINTIES}

4.1 Average Illumination 0.0

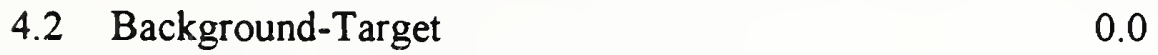

Interactions

4.3 Cross Polarization $\quad 0.0$

4.4 Drift neg.

4.5 Frequency neg.

4.6 Integration n.a.

4.7 I-Q Imbalance neg.

4.8 Near Field neg.

4.9 Noise-Background 0.4

4.10 Nonlinearity $\quad 0.0$

4.11 Range neg.

4.12 Target Orientation $\quad 0.0$

$\begin{array}{lll}4.13 & \text { Calibration Target } & 0.0 \\ 4.14 & \text { Overall Uncertainty (RSS) } & 0.9\end{array}$

Figure 2: A sample table of RCS uncertainties following NIST conventions. See appendix A. 


\section{B Appendix \\ Sphere-Calibration Constant in \\ Dynamic RCS Measurements}

In the main text (see section 4) components of the uncertainty budget for measurement of the calibration target are explained. In the special case of dynamic tracking of a sphere it is the current practice to introduce a spherecalibration constant, determined from data, which is then used to correct the power measurements from other targets. In this appendix we examine the details of computing such a calibration constant with the intent of elucidating how measurement uncertainties enter into this data analysis. In a further study, this information will be fundamental in proposing improvements for this procedure.

The sphere-calibration constant $\rho$ is defined as

$$
\rho \equiv \frac{P_{r}}{P_{r t}},
$$

where $P_{r}$ is the error-free measured received power and $P_{r t}$ is the error-free theoretical received power. This constant, if known for a given measurement system, can be used to convert measured received power to theoretical received power on a dynamic RCS range.

In general, received power $P_{r}$ is expressed as

$$
P_{r}=\frac{P_{t} G_{0}}{4 \pi R^{2}} \cdot \frac{\sigma}{4 \pi R^{2}} \cdot \frac{G_{0} \lambda^{2}}{4 \pi},
$$

where $P_{t}$ is the transmitted power, $R$ is the range, $\sigma$ is the theoretical RCS of the sphere under observation, $\lambda$ is the wavelength at which measurements are being made, and $G_{0}$ is the on-axis gain. All theoretical quantities can be specified without errors, but in practice the measured quantities will have errors in them. Hence, we define error-contaminated quantities $\hat{P}_{r}$, $P_{r t}^{c}, \hat{R}$ and $\hat{G}_{0}$, which represent the measured received power, the calculated theoretical received power, the measured range and the measured on-axis gain, respectively.

The error-contaminated and error-free quantities are related simply by

$$
\begin{gathered}
\hat{P}_{r}=P_{r}+\delta \hat{P}_{r} \\
\hat{R}=R+\delta \hat{R} \\
\hat{G}_{0}=G_{0}+\Delta,
\end{gathered}
$$

where $\delta \hat{P}_{r}, \delta \hat{R}$, and $\Delta$ are the errors in the measured power, measured range, and the measured on-axis gain, respectively. 
We can write the sphere-calibration constant $\rho$ as

$$
\rho \equiv \frac{P_{r}}{P_{r t}} \equiv \frac{P_{r}}{P_{r t}^{c}} \frac{P_{r t}^{c}}{P_{r t}}
$$

to explicitly exhibit the fact that the computed theoretical received power $P_{r t}^{c}$ is different from the exact theoretical received power $P_{r t}$, which cannot be determine from data.

We can use eq (28) to express $P_{r t}^{c}$ in terms of measured quantities, and eq (29) to express $P_{r}$ in terms of measured quantities. Thus,

$$
\frac{P_{r}}{P_{r t}^{c}}=\frac{P_{r}}{\alpha \hat{G}_{0}^{2} / \hat{R}^{4}}=\frac{\hat{P}_{r} \hat{R}^{4}}{\alpha \hat{G}_{0}^{2}}\left(1-\frac{\delta \hat{P}_{r}}{\hat{P}_{r}}\right)
$$

and

$$
\frac{P_{r t}^{c}}{P_{r t}}=\frac{\hat{G}_{0}^{2} / \hat{R}^{4}}{G_{0}^{2} g^{2} / R^{4}}=\frac{1}{g^{2}}\left(1-\frac{\delta \hat{R}}{\hat{R}}\right)^{4}\left(1-\frac{\Delta}{\hat{G}_{0}}\right)^{-2},
$$

where $\alpha \equiv P_{t} \sigma \lambda^{2} /(4 \pi)^{3}$ and $g=g(\theta, \phi)$ defines the receive (transmit) antenna gain function. We can now combine the above expressions to obtain the sphere-calibration constant in terms of measured quantities

$$
\alpha \hat{G}_{0}^{2} g^{2} \rho=\hat{P}_{r} \hat{R}^{4} \epsilon_{P} \epsilon_{R} \epsilon_{G}
$$

where

$$
\begin{aligned}
\epsilon_{P} & =\left(1-\frac{\delta \hat{P}_{r}}{\hat{P}_{r}}\right) \\
\epsilon_{R} & =\left(1-\frac{\delta \hat{R}}{\hat{R}}\right)^{4}
\end{aligned}
$$

and

$$
\epsilon_{G}=\left(1-\frac{\Delta}{\hat{G}_{0}}\right)^{-2} .
$$

In the above derivation we assumed that the quantities $\lambda, P_{t}$ and $g(\theta, \phi)$ are known exactly, or, at least, are constant throughout the measurement. This of course is not correct, and the errors in these quantities can easily be incorporated in eq (33) by including factors such as $\epsilon_{\lambda}, \epsilon_{P_{t}}$, and $\epsilon_{g}$. We will not do so here.

By definition $\rho_{d B}$ is given by

$$
\begin{gathered}
\rho_{d B}=10 \log \left(\hat{P} \hat{R}^{4}\right)-10 \log \left(\alpha \hat{G}_{0}^{2}\right) \\
-20 \log g(\theta, \phi)+\sum_{s=P, R, G} 10 \log \epsilon_{s} .
\end{gathered}
$$


The the first line in eq (35) can yield the calibration constant as it is obtained in current practice; hence, the second line in eq (35) will yield the total error $\Delta \rho \equiv \rho-\rho^{c}$, which is the difference between the exact sphere-calibration constant $\rho$ and $\rho^{c}$, the calibration constant computed from data.

To obtain the sphere-calibration constant, we average eq (35) over a large number of measurements, as the received power is measured as a function of measured range and time. Hence, the averaging is usually over a range of decibels, which in practice is as large as $10 \mathrm{~dB}$. We get the measured calibration constant $\left\langle\rho_{d B}\right\rangle$ as

$$
\left\langle\rho_{d B}\right\rangle=10\left\langle\log \left(\hat{P}_{r} \hat{R}^{4}\right)\right\rangle-10 \log \left(\alpha \hat{G}_{0}^{2}\right),
$$

and the error $\Delta \rho$ is

$$
\Delta \rho=-20\langle\log [g(\theta, \phi)]\rangle+\left\langle\sum_{s=P, R, G} 10 \log \epsilon_{s}\right\rangle .
$$

We make the following observations:

(a) $\epsilon_{R} \approx 0.013 \mathrm{~dB}$ is small (assuming $\delta \hat{R} \approx 2-10 \mathrm{~m}$ );

(b) $\epsilon_{G}$ is an unknown constant offset present in all measurements, and, hence, will not introduce an error in measurements on targets;

(c) the beam pointing error term $20\langle\log g(\theta, \phi)\rangle$ is unknown, presumably small, but could be large in a dynamic tracking system;

(d) $\epsilon_{P}$ can be significant for $S / N<30 \mathrm{~dB}$.

The terms expressing the beam-pointing error and the error in the measured power deserve further examination.

In the main text an estimate of the beam pointing error was given for a $\cos ^{2}$ beam shape. Alternatively, we can model the beam in one dimension as

$$
g(\theta)=10^{-\beta \theta^{2} / \theta_{0}^{2}}
$$

where $\beta=\log 2$, and $2 \theta_{0}$ is the $3-\mathrm{dB}$ beamwidth. It is easy to show that for a uniform distribution of $\theta$ the average of the beam pointing error for this model is

$$
-20\langle\log [g(\theta)]\rangle \approx 2 \frac{\theta_{M}^{2}}{\theta_{0}^{2}}
$$

where $\theta_{M}$ is the maximum beam pointing error. This can be significant for narrow beams!

Since, in practice, sphere-calibration measurements are taken over a few thousand meters as the calibration sphere is moving with the wind, the received power varies over approximately a $10 \mathrm{~dB}$ range. Since $S / N$, the signal-to-noise ratio, varies inversely with the range, the factor $\left\langle\hat{P}_{r} \hat{R}^{4}\right\rangle$ in the computed sphere-calibration constant favors weaker signals, thereby introducing errors in the sphere-calibration constant that could be eliminated 
by alternative calibration procedures. This will be examined under a separate study.

\section{Appendix RCS Frequency Dependence}

In the main text (see section 3.5) we discuss the component of uncertainty in RCS due to uncertainty in frequency. There, we mention that target RCS, standard RCS, and system gain all depend implicitly on frequency. Here, we examine examples of these dependencies.

Consider the situation where a small calibration target is used. If the target were a perfectly conducting ellipsoid or a squat cylinder, then its RCS would be proportional to $f_{s}^{4}$ [4]. The antenna gain, in the case of a parabolic reflector or a horn, is proportional to $f_{s}^{2}[5]$. Thus from (7), a frequency uncertainty $\Delta f_{s}$ gives

$$
\Delta \sigma(\mathrm{dB})=-60 \log \left(1-\frac{\Delta f_{s}}{f_{s}}\right),
$$

which may be compared to eq (23). If $\Delta f_{s} / f_{s}=0.2 \%$, we have $\Delta \sigma(\mathrm{dB})$ $=0.05$.

Next, consider two identical point scatterers which are separated by a down-range distance $L$. Their individual responses will be $4 \pi L / \lambda$ radians apart in phase ( $\lambda$ is the wavelength). A frequency uncertainty $\Delta f / f=$ $\lambda /(2 L)$ is equivalent to a $360^{\circ}$ uncertainty in relative phase. For point scatterers separated by $125 \lambda$, a $0.2 \%$ frequency error will produce a $180^{\circ}$ relative phase change, and could cause the combined response to vary from a maximum to a null. 


\section{References}

[1] M. W. Whitt, F. T. Ulaby, P. Polatin, and V. V. Liepa, "A general polarimetric radar calibration technique," IEEE Trans. Antennas Propagat., vol. AP-39, pp. 62-67, Jan. 1991.

[2] B. N. Taylor, and C. E. Kuyatt, "Guidelines for evaluating and expressing the uncertainty of NIST measurement results," National Institute of Standards and Technology Technical Note 1297, Jan. 1993.

[3] S. J. Press, Bayesian Statistics. Wiley: New York, 1988.

[4] G. T. Ruck, Radar Cross Section Handbook, Vol. 1. Plenum Press: NY, 1970.

[5] C. A. Balanis, Antenna Theory. Harper \& Row: NY, 1982. 

